\title{
¿Qué cualidades valoran más los Pacientes de su Médico de Familia?
}

\author{
Miriam Motilla Frailea, Raquel Sánchez-Oro Gómeza, Olga Curiel Sainz ${ }^{\mathrm{a}}$, \\ Belén Rodríguez Macías ${ }^{a}, M^{a}$ Jesús López Gila ${ }^{a}$ Francisco López de Castro ${ }^{b}$
}

\begin{abstract}
${ }^{a}$ Residente de Medicina Familiar y Comunitaria. Unidad Docente de Toledo

${ }^{\mathrm{b}}$ Coordinador de la Unidad Docente de Medicina Familiar y Comunitaria de Toledo.
\end{abstract}

\section{Correspondencia: Francisco} López de Castro, Unidad Docente de Toledo, c/ Barcelona 2, 45005-Toledo, España.

Teléfono: 0034- 925259908.

Email: flopez@sescam.org

Recibido el 4 de diciembre de 2008.

Aceptado para su publicación el 26 de diciembre de 2008.

\section{RESUMEN}

Objetivo. Conocer qué cualidades del Médico de Familia son más valoradas por los pacientes y en qué medida son cumplidas.

Diseño. Estudio descriptivo transversal.

Emplazamiento. Atención Primaria. Área de Toledo.

Participantes. Mayores de 14 años del Área, seleccionados por muestreo aleatorio sistemático.

Mediciones principales. Encuesta telefónica diseñada ad hoc con ítems sobre las características relevantes del médico y el cumplimiento de las mismas, en escala de 0 a 10 . Se recogieron también variables sociodemográficas, enfermedades crónicas y utilización de consultas.

Resultados. Fueron entrevistados 161 pacientes, con edad media de 42,6 años. El 55,7\% mujeres. El 69,7\% tenía alguna enfermedad crónica. El 38,6\% de ellos había acudido a su médico en el último mes, mientras que el $90,4 \%$ lo había hecho en el último año. Las características más valoradas fueron la 'confiabilidad' y el 'trato respetuoso'; las menos valoradas fueron la 'participación en la toma de decisiones' y la 'aceptación de pruebas demandadas por el paciente'. Respecto al grado de cumplimiento de dichas cualidades, destacan el 'trato respetuoso' y la 'confiabilidad'.

Conclusiones. La utilización de consulta es muy elevada en nuestro medio. Los pacientes valoran más cualidades de "índole personal" del médico que las de tipo técnico u organizativo. La participación en la toma de decisiones es de las menos valoradas. En general, los médicos del Área de Toledo cumplen en buena medida las expectativas planteadas en lo que a las cualidades personales se refiere.

Palabras clave. Satisfacción del Paciente. Medicina Familiar y Comunitaria.

\section{ABSTRACT}

What qualities do patients value more in a Family Doctor?

Objective. To determine what qualities patients value most in a Family Doctor and to what extent their doctors meet these expectations.

Design. Cross sectional, descriptive study.

Setting. Primary care. Toledo.

Participants. Patients over 14 years old, selected by a random sampling method.

Main measurements. An ad hoc telephone survey with items on the characteristics of a doctor and compliance to them, measured on a scale of 1 to 10. Socio-demographic variables, chronic diseases and use of doctors' surgeries were also recorded.

Results. A total of 161 patients with a mean age of 42.5 years were interviewed. $55.7 \%$ were women. $69,7 \%$ had a chronic disease. $38,6 \%$ had visited their doctor in the last month, whilst $90.4 \%$ had done so in the last year. The most valued characteristics were 'reliability' and 'respectful treatment'; the least valued were "participation in making decisions" and "agreeing to tests requested by the patients". "Respectful treatment" and "reliability" were the characteristics that most met the patients' expectations.

Conclusions. The use of doctors' surgeries is very high in our area. The patients valued qualities of a "personal nature" more than technical or organisational qualities. Participation in the decision making process was less valued. In general doctors in the Toledo health area met patients' expectation as regards personal characteristics.

Key words. Patient Satisfaction. Family Practice. 


\section{INTRODUCCIÓN}

A lo largo de la historia del ejercicio médico, el papel del paciente se ha ido transformando desde una actitud expectante ante el paternalismo de su médico, que tomaba las decisiones en todo momento, hasta la situación actual en la cual el paciente se ha convertido en un sujeto más activo en la relación médico-paciente, asumiendo un mayor protagonismo en el control del proceso ${ }^{1}$. En este cambio han influido en gran medida la evolución de los sistemas de información y la tecnología, que permiten que los usuarios/pacientes dispongan de una información actualizada y mayor sobre los procesos que les conciernen². Nuestro "nuevo paciente" quiere participar en la toma de decisiones que incumben a su salud, tanto en aspectos del proceso diagnóstico, por ejemplo la solicitud de pruebas complementarias, como en el proceso terapéutico ${ }^{3}$.

En paralelo, este paciente, más activo y participativo, demanda una atención sanitaria de mayor calidad, requiriendo de los profesionales sanitarios (especialmente de su Médico de Familia) una serie de cualidades que van más allá de las meras habilidades técnicas. El paciente espera de su médico honestidad, integridad, respeto, responsabilidad, compromiso, etc ${ }^{4}$. La relación médico-paciente se convierte así en un 'encuentro entre personas' del que depende en gran medida el grado de satisfacción del paciente, en la actualidad uno de los principales indicadores de la calidad de la atención sanitaria prestada 5 .

Este asunto ha sido un tema estudiado y debatido frecuentemente en la literatura científica de los últimos años, concluyéndose en un gran número de estudios, de países tales como Reino Unido, EEUU, Suecia o Méjico ${ }^{1-8}$, que cualidades tales como empatía, atención personalizada, comprensión, información detaIlada, etc. por parte del profesional sanitario traducen finalmente una mejora en la realización de actividades preventivas, en un mejor seguimiento de los procesos crónicos y en un mayor cumplimiento terapéutico, al conseguir una mayor satisfacción y confianza por parte de los pacientes.

En este contexto, el objetivo del presente estudio fue conocer qué cualidades del Médico de Familia son más valoradas por los pacientes en nuestro medio y en que medida son cumplidas por aquéllos.

\section{MATERIAL Y MÉTODO}

Se trata de un estudio descriptivo, de corte transversal. La población diana fueron todas las personas mayores de 14 años adscritas a algún médico de familia del Área de Salud de Toledo, que cubre una población de aproximadamente 400.000 habitantes. Se excluyeron del estudio a aquellas personas con deterioro cognitivo o no hispanoparlantes.

El muestreo se realizó de forma aleatoria sistematizada, utilizando como base de datos el listado de tarjetas sanitarias del Área de Salud de Toledo facilitado por la Gerencia de Atención Primaria de Toledo. El cálculo del tamaño muestral se hizo para estimar la puntuación de cada ítem (en una escala de 0 a 10 puntos) con una seguridad del $95 \%$ y una precisión de $\pm 0,25$ puntos, asumiendo una desviación típica en torno a 1,5 puntos, resultando suficiente un tamaño de 150 individuos. En previsión de posibles problemas (errores en el listado, no localización, no respuestas, etc.) se solicitó un listado de 400 individuos.

La recogida de datos se realizó durante los meses de febrero, marzo y abril, mediante entrevista telefónica, por los propios investigadores. Los entrevistadores fueron previamente entrenados y se realizo un pilotaje inicial para detectar posibles problemas. Aquellos casos en los que no se pudo localizar a la persona seleccionada tras tres llamadas telefónicas realizadas a diferentes horas o días, fueron considerados ausentes.

Para valorar la opinión del usuario se diseñó una encuesta ad hoc (tabla 1) cuyos ítems recogían aspectos que, según la bibliografía consultada ${ }^{9-14}$, se consideraron como características relevantes que debería presentar un médico. En cada uno de los ítems se valoraba el grado en que el paciente lo consideraba como importante y el grado en que el paciente consideraba que su médico cumplía dicha característica; en ambos casos valorado según una escala numérica de 0 a 10 donde el 0 significaba "ninguna importancia" o "no lo cumple en absoluto" y el 10 "de máxima importancia" o "lo cumple siempre".

La entrevista también recogía una serie de variables sociodemográficas como la edad, el sexo, el estado civil, el nivel de estudios, diferenciando 'sin estudios', 'primarios' (hasta $8^{\circ}$ EGB ó $4^{\circ}$ de la ESO), 'secundarios' (bachiller o Formación Profesional) y 'universitarios', la localidad de residencia, según número de habitantes de su localidad $(<1.000,1.000-5.000$, $5.000-10.000$ y $>10.000$ ) y la presencia de enfermedades crónicas. También se interrogaba sobre la utilización de la consulta de su médico de familia en el último año.

Los datos fueron introducidos en una base de datos creada al efecto y analizados con el programa estadístico SPSS 12.0 para Windows, empleando herra- 
mientas de estadística descriptiva (porcentaje, media y desviación típica) y analítica ( $U$ de Mann-Whitney para comparación de medias).

\section{RESULTADOS}

Finalmente se llegó a contactar con 216 individuos, de los cuales aceptaron participar 161 (74,5\%). La edad media de los participantes fue de 42,6 años (DE 16,6). El $55,7 \%$ fueron mujeres. El $17,7 \%$ no tenía estudios, el $38,6 \%$ primarios, el $27,8 \%$ secundarios y el $15,8 \%$ universitarios. El 58,9\% estaban casados o emparejados. El $45,6 \%$ de ellos residía en una localidad de más de 10.000 habitantes, el resto en zona rural.

El 69,7\% tenía alguna enfermedad crónica. El 38,6\% de ellos había acudido a su médico en el último mes,

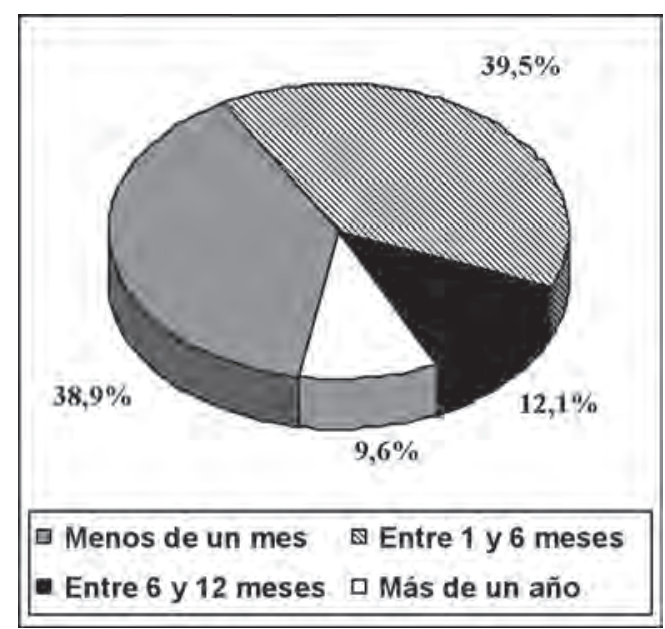

Figura 1. Tiempo transcurrido desde la última visita al médico. mientras que el 90,4\% lo había hecho en el último año (IC 95\%: 84,1-94,0\%] (figura 1).

En la figura 2 se muestran las valoraciones otorgadas a cada cualidad de los médicos de familia en general. Las características más valoradas fueron la 'confiabilidad' $(9,4$ puntos sobre 10) y el 'trato respetuoso' $(9,3)$, mientras que las menos valoradas fueron la 'participación en la toma de decisiones' $(7,9)$ y la 'aceptación de pruebas demandadas por el paciente' $(8,0)$.

Al analizar este perfil según sexo, edad y presencia de enfermedades crónicas (tabla 2), se observan pequeñas diferencias en algunos ítems, aunque en general se mantiene el orden de valoración. El análisis por nivel de estudios, hábitat y estado civil tampoco encontró diferencias importantes.

Respecto al grado de cumplimiento de dichas cualidades observado por los pacientes en su propio médico (figura 3), destacan el 'trato respetuoso' (8,7 sobre 10) y 'confiabilidad' $(8,3)$, mientras que los que menor grado de cumplimiento tienen son el 'cumplimiento de horarios' $(6,4)$ y la 'participación en la toma de decisiones $(7,1)$.

\section{DISCUSIÓN}

Antes de entrar a discutir los resultados obtenidos en este estudio, es obligado mencionar que la tasa de no-respuestas del $25 \%$, aunque similar a la encontrada en otras encuestas telefónicas, puede suponer un pequeño sesgo en la valoración del grado de cumplimiento de las cualidades de los médicos de familia

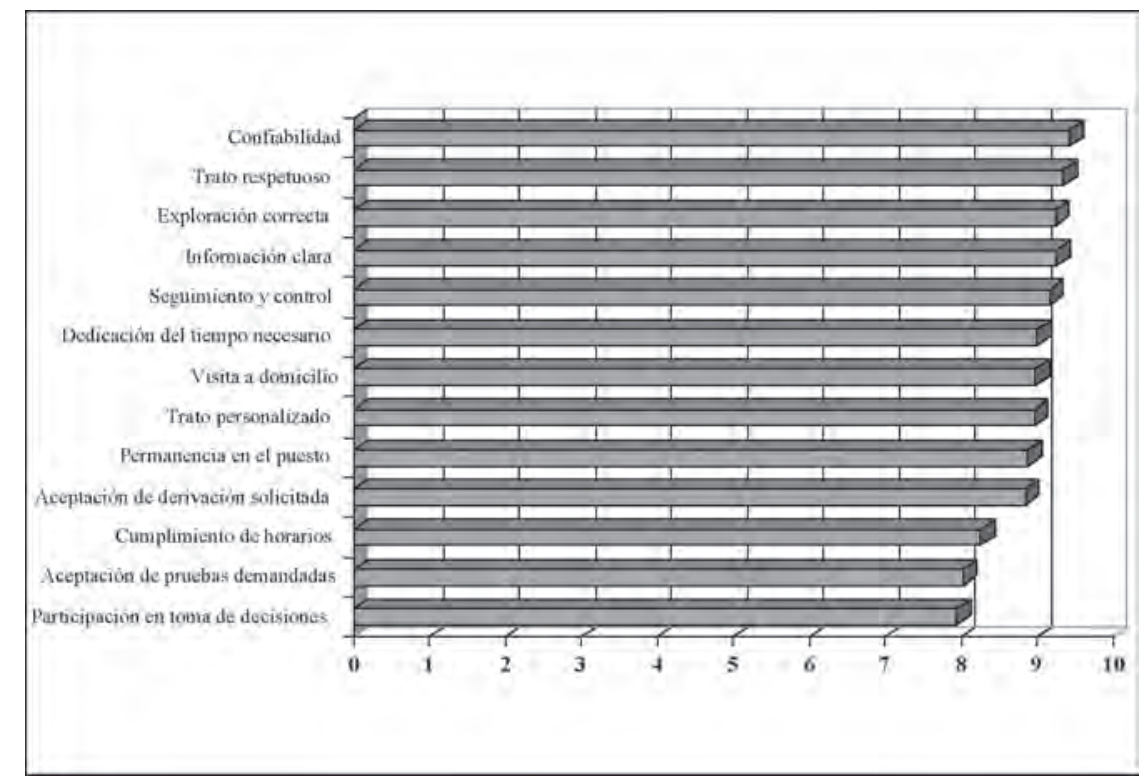

Figura 2. Valoración de distintas cualidades del Médico de Familia. 


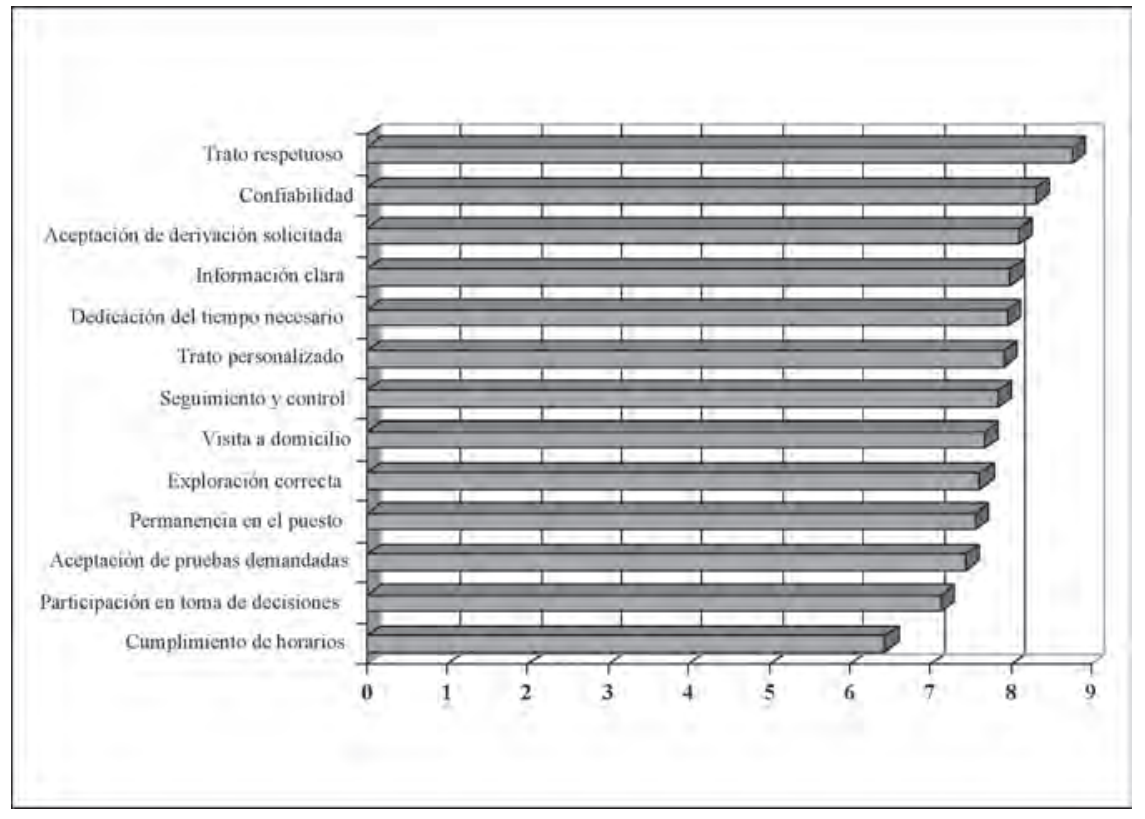

Figura 3. Grado de cumplimiento de las cualidades de su propio Médico de Familia.

si la verdadera razón de la no participación estuviera relacionada con una "valoración negativa de su médico" (la mayoría de las razones dadas para no colaborar fueron por "no disponer de tiempo").

Un primer dato que nos llamó la atención fue el altísimo porcentaje de utilización de consultas ("extensión de uso"), tanto en el último mes (icasi un $40 \%$ !) como en el último año (¡más del $90 \%$ !). En las últimas Encuestas de Salud de nuestra Comunidad Autónoma, la utilización de consultas de Atención Primaria por adultos era del $68,2 \%$ en 2002 y del $76 \%$ en 2006 , lo que sugiere un incremento constante en los últimos años ${ }^{15,16}$. Además, considerando la edad media de nuestra muestra (42,6 años), relativamente joven, resulta aún más llamativo. Creemos que se debería profundizar en este asunto y analizar las verdaderas razones de esta demanda de servicios tan elevada en Atención Primaria, ya que el nivel de salud de nuestra población no nos parece explicación suficiente para ello.

En cuanto a la valoración general de la importancia de las cualidades demandadas al médico de familia, la puntuación en todos los ítems es elevada, lo que refleja un alto nivel de exigencia de nuestros pacientes. Ocupan los primeros puestos de este ranking cualidades de "índole personal" del médico, tales como la confiabilidad, el trato respetuoso o la información, por encima de otras más técnicas (exploración, seguimiento y control) u organizativas (visita a domicilio, cumplimiento de horarios, permanencia en la plaza). Los pacientes priorizan en el médico cualidades que respeten sus derechos (a un trato digno, a la infor- mación, a la confidencialidad) ${ }^{17}$; quieren que un buen médico sea ante todo una buena persona que les resulte confiable, antes que un técnico experto e infalible (si es que esto fuera posible) ${ }^{18}$.

Curiosamente, el ítem menos valorado por los pacientes ha sido la participación en la toma de decisiones, cuestión a la que en nuestra sociedad y en los medios de comunicación y políticos se está dando mucha importancia ${ }^{19}$, pero que no termina de hacerse realidad en nuestras consultas ${ }^{20}$.

Si extrapolamos esas cualidades demandadas al grado en que sus médicos de familia cumplían esas expectativas, se observó que las dos cualidades que los usuarios del Área de Toledo consideraron como las más importantes en todo médico de familia (confiabilidad y trato respetuoso), a la hora de evaluar a sus propios médicos resultaron ser esas dos mismas cualidades las de mayor cumplimiento, lo cual lleva a pensar que los médicos del Área de Toledo cumplen en buena medida las expectativas planteadas en lo que a las cualidades personales se refiere. Aunque, como se dijo antes, cabe la posibilidad de un sesgo en este sentido por la tasa de no-respuestas habida.

No ocurre igual con cuestiones organizativas, en especial con el cumplimiento de horarios, en las que creemos que existe un margen de mejora importante, pero que no suele deberse a factores dependientes exclusivamente del médico.

Obviamente, las valoraciones de algunos ítems varían dependiendo de las circunstancias especiales de 
Le vamos a enunciar una serie de características que consideramos que debería reunir un médico de atención primaria. Primero puntúe de 0 a 10 (siendo 0 nada importante y 10 muy importante) la importancia que usted le concede a cada una de esas cualidades. Luego, valore de 0 a 10 en qué medida su propio médico cumple esa cualidad, entendiendo que 0 significa que no lo cumple nunca y 10 que lo cumple siempre.

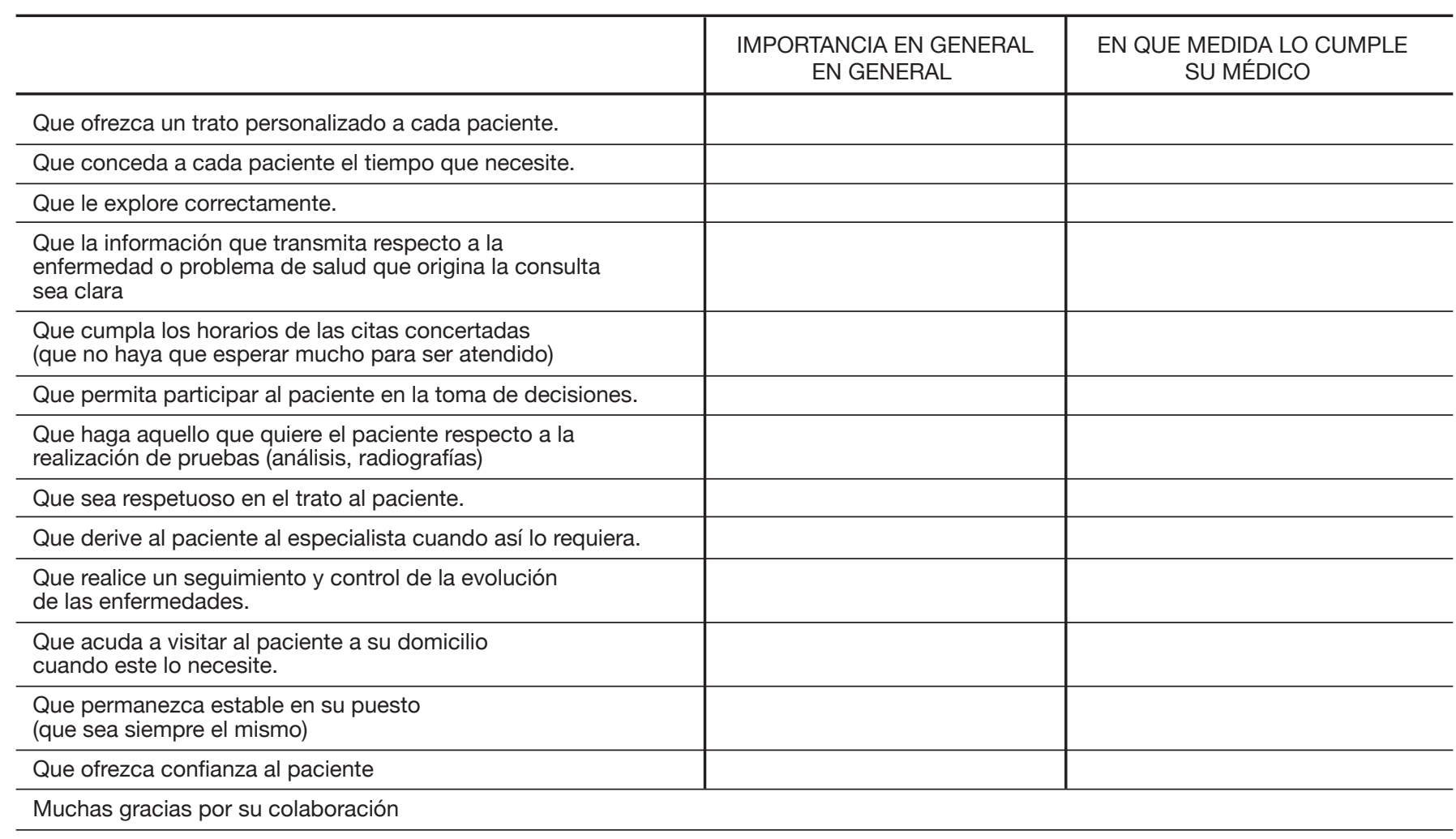

Tabla 1. Encuesta tipo.

\begin{tabular}{|c|c|c|c|c|c|c|c|c|c|}
\hline \multirow[t]{2}{*}{ CUALIDAD } & \multirow[b]{2}{*}{ Hombres } & \multirow{2}{*}{$\begin{array}{l}\text { SEXO } \\
\text { Mujeres }\end{array}$} & \multirow[b]{2}{*}{$\mathrm{p}$} & \multicolumn{2}{|c|}{ EDAD } & \multirow[b]{2}{*}{$\mathrm{p}$} & \multicolumn{3}{|c|}{$\begin{array}{l}\text { ENFERMEDADES } \\
\text { CRÓNICAS }\end{array}$} \\
\hline & & & & $<40$ años & $>40$ años & & No & Sí & $\mathrm{p}$ \\
\hline Trato personalizado & 8.9 & 8.9 & $>0,1$ & 8.9 & 8.9 & $>0,1$ & 8.9 & 9.1 & $>0,1$ \\
\hline Dedicación del tiempo necesario & 8.9 & 9.0 & $>0,1$ & 9.0 & 8.9 & $>0,1$ & 9.0 & 9.0 & $>0,1$ \\
\hline Exploración correcta & 9.2 & 9.2 & $>0,1$ & 9.5 & 8.9 & $<0,05$ & 9.3 & 9.1 & $>0,1$ \\
\hline Información clara & 9.0 & 9.3 & $>0,1$ & 9.2 & 9.1 & $>0,1$ & 9.2 & 9.2 & $>0,1$ \\
\hline Cumplimiento de horarios & 8.2 & 8.2 & $>0,1$ & 8.1 & 8.3 & $>0,1$ & 8.2 & 8.0 & $>0,1$ \\
\hline Participación en toma de decisiones & 7.6 & 8.1 & $>0,1$ & 7.7 & 8.1 & $>0,1$ & 7.7 & 8.3 & $>0,1$ \\
\hline Aceptación de pruebas demandadas & 8.1 & 7.9 & $>0,1$ & 8.0 & 8.0 & $>0,1$ & 7.8 & 8.3 & $>0,1$ \\
\hline Trato respetuoso & 9.2 & 9.4 & $>0,1$ & 9.4 & 9.2 & $>0,1$ & 9.3 & 9.4 & $>0,1$ \\
\hline Aceptación de derivación solicitada & 8.7 & 8.9 & $>0,1$ & 8.9 & 8.7 & $>0,1$ & 8.6 & 9.0 & $>0,1$ \\
\hline Seguimiento y control & 8.9 & 9.3 & $<0,1$ & 9.2 & 9.1 & $>0,1$ & 9.0 & 9.3 & $>0,1$ \\
\hline Visita a domicilio & 8.6 & 9.2 & $<0,05$ & 8.9 & 9.0 & $<0,1$ & 8.7 & 9.4 & $<0,05$ \\
\hline Permanencia en el puesto & 8.6 & 9.0 & $<0,1$ & 8.6 & 9.0 & $<0,05$ & 8.6 & 9.4 & $<0,05$ \\
\hline Confiabilidad & 9.2 & 9.5 & $<0,1$ & 9.3 & 9.4 & $>0,1$ & 9.3 & 9.5 & $>0,1$ \\
\hline
\end{tabular}

Tabla 2. Valoración de las cualidades del Médico de Familia según edad, sexo y presencia de enfermedades crónicas. 
cada paciente. Así, por ejemplo, la permanencia en el puesto o la visita a domicilio son más valorados por los pacientes con enfermedades crónicas o los mayores de cuarenta años, que desean ser asistidos siempre por la misma persona y a ser posible en su domicilio, a veces por ver mermada su capacidad de movilidad.

La verdadera relación médico-paciente ha consistido siempre en un contrato cuya validez reside en un intercambio emocional entre medico y paciente. Dicho contrato defiende, por un lado, las expectativas del paciente de que su médico sea bueno y actúe lo mejor posible, mientras que por el otro lado se cede al médico la posibilidad de desarrollar su profesión de forma autónoma sin apenas control externo. Este contrato ha sido válido hasta el momento en que en la sociedad ha aparecido un modelo de paciente más activo, el cual dispone de mayor información, y de ahí que quiera exigir explicaciones razonadas respecto a su padecer. Si no queda satisfecho, solicitará segundas opiniones y finalmente, dado que se trata de su bien más preciado, su propia persona, querrá participar en la toma de decisiones ${ }^{7}$. A causa de esto, cada día es más necesario un cambio radical en la forma de actuar del médico, y por tanto una variación de ese "contrato", ya que la situación actual a la que se ve sometida un médico (mayor demanda asistencial y menor tolerancia a los errores), unido al nuevo modelo "activo" de paciente, hacen que dicha relación médico-paciente se vea desequilibrada por parte de la balanza médica.

Con ello, decir que todo médico habría de prepararse en una importante aptitud: desarrollar una relación simétrica emocionalmente con sus pacientes. Esperemos ir progresando en ello; creemos que trabajos de este tipo pueden hacernos reflexionar sobre las expectativas del paciente y mejorar la atención que prestamos.

\section{BIBLIOGRAFÍA}

1- Torres Arreola LP, Doubova SV, Reyes Morales H, Villa Barragan JP, Constantino Casas P, Pérez Cuevas R. Estudio de las necesidades de salud en atención primaria mediante el diagnóstico de salud de la familia. Aten Primaria 2006; 38(7):381-6.

2- Ortiz E, Clancy CM. Use of information technology to improve the quality of health care in the United States. Health Serv Res
2003; 38(2):xi-xxii.

3- Little P, Dorward M, Warner G, Stephens K, Senior J, Moore $M$. Importance of patient pressure and perceived pressure and perceived medical need for investigations, referral, and prescribing in primary care: nested observational study. BMJ 2004; 328:444.

4- Jovell A. Medicina basada en los pacientes. ¿Quién y cómo hay que formar a los médicos? Jano 2008; 1701:46-50.

5- Wingart SN, Pagovich O, Sands DZ, Li JM, Aronson MD, Davis RB et al. Patient-reported service quality on a medicine unit. Int J Qual Health Care 2006; 18(2):95-101.

6- Andén A, Andersson SO, Rudebeck CE. Satisfaction is not allpatients'perceptions of outcome of general practice consultations, a qualitative study. BMC Family Practice. 2005; 6:43. Disponible en URL: http://www.biomedcentral.com/content/ pdf/1471-2296-6-43.pdf [consultado el 10/10/2008].

7- Wein S. Autonomy, paternalism, and the doctor's duty of care. Palliat Support Care. 2006; 4(4):331-2.

8- Mercer SW, McConnachie A, Maxwell M, Heaney D, Watt G. Relevance and practical use of the Consultation and Relational Empathy (CARE) Measure in general practice. The Author (2005). Published by Oxford University Press. Family Practice Advance originally published online on 16 March 2005.

9- McKinstry B, Colthart L, Walker J. Can doctors predict patients'satisfaction and enablement? A cross-sectional observational study. The Author (2006). Published by Oxford University Press. Family Practice Advance Access published on 3 February 2006.

10- Mercer SW, Reynolds W. Empathy and quality of care. Br J Gen Pract 2002; 52(Suppl):S9-S12.

11- Wall G. The inverse care law today. Lancet 2002; 360:252-4.

12- Streiner DL, Norman GR. Health measurement scales (third edition). Oxford: Oxford University Press; 2003.

13- Jung HP, Wensing M, Grol R. What makes a good general practitioner: do patients and doctors have different views? $\mathrm{Br} J$ Gen Pract 1997; 47:805-9.

14- Kremer D. Quality at general practice consulations. BMJ 2000; 320:315.

15- Observatorio de Salud FISCAM. Encuesta de Salud 2006. Disponible en: http://www.jccm.es/sanidad/FISCAM/ensa06/ adultos/ADUL_5_UTILIZACIONSERVICIOS.pdf. [consultado el 28-08-2008].

16- Encuesta de Salud de Castilla La Mancha 2002. Disponible en: http://www.jccm.es/sanidad/fiscam/upload/Utilizacion\%20de \%20servicios_i.pdf [consultado el 28-08-2008].

17- Navarro Rubio MD, Gabriele Muñiz G, Jovell Fernández AJ. Los derechos del paciente en perspectiva. Aten Primaria 2008; 40(7):367-9.

18- Jovell AJ, Navarro Rubio MD, Fernández Maldonado L, Blancafort S. Nuevo rol del paciente en el sistema sanitario. Aten Primaria 2006; 38(3):234-7.

19- Barca Fernández I, Parejo Mínguez R, Gutiérrez P, Fernández Alarcón F, Alejandre Lázaro G, López de Castro F. La información al paciente y su participación en la toma de decisiones clínicas. Aten Primaria 2004; 33(7):361-7.

20- Tardáguila Lobato MP, Méndez Pérez CL, Torres Vélez JC, Méndez García T, Benito Martín B y Lopez de Castro F. La información al paciente y su participación en la toma de decisiones en las urgencias de Atención Primaria. Rev Clin Med Fam 2008; 2(3):101-5. 